Conclusions Severe ROP remains a strong marker of childhood disabilities in a recent and large international cohort of infants $\leq 1250$ g BW.

\section{THE ASSOCIATION BETWEEN PRETERM BIRTH AND AUTISM COULD BE EXPLAINED BY MATERNAL AND NEONATAL MORBIDITY}

doi:10.1136/archdischild-2012-302724.0330

S Johansson, S Buchmayer, A Johansson, C Hultman, P Sparen, S Cnattingius. Karolinska Institutet, Stockholm, Sweden

Background and Aims Children born preterm face an increased risk of autistic disorders.

We examined whether the association between preterm birth and risk of autistic disorders could be explained by pregnancy complications or neonatal morbidity.

Methods Swedish, population-based, case-control study including 1216 cases with autistic disorders born between 1987 and 2002, and 6080 controls matched with respect to gender, birth year, and birth hospital. Associations between gestational age and autistic disorders were assessed and adjusted for maternal, birth and neonatal characteristics.

Results Compared with infants born at term, the unadjusted odds ratios (ORs) for autistic disorders among very and moderately preterm infants were 2.05 [95\% CI: $1.26-3.34$ ] and 1.55 [95\% CI: 1.22 $1.96]$, respectively.

In analyses controlled for maternal, pregnancy, and birth characteristics, ORs were reduced to 1.48 [95\% CI: 0.77-2.84] and 1.33 [95\% CI: 0.98-1.81], respectively.

Adding also neonatal complications to the analyses, ORs were 0.98 [95\% CI: 0.45-2.16] and 1.25 [95\% CI: 0.90-1.75], respectively.

Reductions in risks of autistic disorders related to preterm birth were primarily attributable to preeclampsia, small-for-gestational age birth, congenital malformations, low Apgar scores at 5 minutes, and intracranial bleeding, cerebral edema, or seizures in the neonatal period. Neonatal hypoglycemia, respiratory distress, and neonatal jaundice were associated with increased risk of autistic disorders for term but not preterm infants.

Conclusions The increased risk of autistic disorders related to preterm birth is mediated primarily by prenatal and neonatal complications that occur more commonly among preterm infants.

\section{THE PROGNOSTIC VALUE OF AEEG AND NIRS DURING THERAPEUTIC HYPOTHERMIA IN TERM ASPHYXIATED NEWBORNS}

doi:10.1136/archdischild-2012-302724.0331

${ }^{1} \mathrm{C}$ Niezen, ${ }^{1} \mathrm{~A}$ Bos, ${ }^{2} \mathrm{D}$ Sival, ${ }^{1} \mathrm{H}$ ter Horst. ${ }^{1}$ Pediatrics; ${ }^{2}$ Child Neurology, University Medical Center Groningen, Groningen, The Netherlands

Background and objective Infants with hypoxic-ischemic encephalopathy (HIE) are treated with therapeutic hypothermia (HT). Following perinatal asphyxia amplitude-integrated EEG (aEEG) and near-infrared spectroscopy (NIRS) are used to determine prognosis. We aimed to assess the prognostic value of aEEG and NIRS during HT.

Methods 40 term infants with HIE and treatment with HT were retrospectively studied. aEEG and NIRS were started immediately following admission. aEEGs were assessed by pattern recognition: background pattern (BP), presence of sleep wake cycling (SWC) and epileptic activity (EA) were appraised. Recordings during HT (72 hrs) were analysed.

Results $84 \%$ of infants had an abnormal BP (discontinuous normal voltage, burst suppression (BS), continuous low voltage (CLV) or flat trace $(\mathrm{FT}))$ at admission. The $\mathrm{LR}+(95 \% \mathrm{CI})$ of an severely abnormal BP (BS, CLV, FT) for mortality was 1.97 (1.24-3.12) at $6 \mathrm{~h}$ after birth and increased to 4.5 (3.16-6.39) at 24h, $6.3(2.04-19.4)$ at $48 \mathrm{~h}$ and 6.19 (1.93-19.8) at 72h. LR+ of BS for mortality was below 1 at any time. LR+ of EA for mortality was 4.95 (2.20-11.1), the type of EA (e.g. status epilepticus) was not predictive. LR+ of SWC for survival was 10.7 (1.62-70). RcSO2 increased from 6 to $72 \mathrm{~h}$ after birth, but was not different at any time between infants that died or survived.

Conclusion aEEG during HT can still be used to predict risk for mortality of HIE, especially beyond $24 \mathrm{hrs}$. BS is frequently not associated with a fatal outcome. RcSO2 has no additional value to predict mortality.

\section{THE RELATIONSHIP BETWEEN RAPID FLUCTUATION IN SERUM SODIUM AND INTRAVENTRICULAR HEMORRHAGE (IVH) IN HYPERNATREMIC EXTREMELY LOW BIRTH WEIGHT PRETERM INFANTS}

doi:10.1136/archdischild-2012-302724.0332

S Sarkar, J Dalton, R Dechert. Department of Pediatrics, Division of Neonatal-Perinatal Medicine, University of Michigan Health System, Ann Arbor, MI, USA

Hypernatremia causes brain shrinkage and resultant vascular rupture with cerebral and IVH. However, it is not known if rapid fluctuation in serum sodium in hypernatremic preterm infants results in IVH or death

Objective To determine if the rapid rise in serum sodium or rapid correction of hypernatremia predict the composite outcome of severe IVH (grade 3 and 4) or death during the first 10 days of life.

Methods Single center retrospective review of 167 preterm infants with GA $\leq 26$ weeks who had serum sodium monitored at least every 12-24 hours and more frequently, if indicated. Logistic regression analysis identified which of the commonly cited risk factors of $\mathrm{IVH}$, including rapid ( $>10$ and $>15 \mathrm{mmol} / \mathrm{l} /$ day) rise or fall in serum sodium could predict composite outcome in hypernatremic infants. Results 98 (59\%) of 167 infants studied developed hypernatremia (serum sodium $>150 \mathrm{mmol} / \mathrm{L}$ ), with a maximum median serum sodium of $154 \mathrm{mmol} / \mathrm{l}$ (range 150-181, IOR 152-157), occurring on median postnatal age of 4 days (IOR 3-5). Grade 4 IVH was more frequent in hypernatremic compared to normonatremic infants $(p=0.032$, OR 3.4, 95\% CI 1.1-10.6). Among 98 infants with hypernatremia, severe IVH or death occurred in 33 and 21 infants with rapid ( $>10 \mathrm{mmol} / \mathrm{l} /$ day) rise and drop in serum sodium, respectively. However, rapid ( $>10$ and $>15 \mathrm{mmol} / \mathrm{l} /$ day) rise or fall in serum sodium was not associated with composite outcome on multivariate analysis.

Conclusion Correction of hypernatremia not exceeding 10 to 15 $\mathrm{mmol} / \mathrm{l} /$ day in hypernatremic preterm infants was not associated with severe IVH or death.

\section{AUTOMATIC IDENTIFICATION OF ACTIVITY BURSTS IN EEG OF PRETERM INFANTS}

doi:10.1136/archdischild-2012-302724.0333

K Murphy, R Goulding, N Stevenson, GB Boylan. Paediatrics and Child Health, University College Cork, Cork, Ireland

Background EEG monitoring provides important information about the neurological status of the preterm infant but is difficult to interpret for most. We aim to automatically detect the typical bursting pattern (trace discontinu) of the preterm EEG and compare the detections with expert manual annotations.

Methods The method was based on the single channel EEG method of Palmu et al. but extended to 8-channel recordings for the first time. The EEG signal was first filtered with a Kaiser-window filter and the output of a non-linear energy operator (NLEO) was calculated. The NLEO signal was smoothed and corrected for baseline artefacts. A burst was identified if the resulting signal remained 